\title{
Dasch Discovery of a Possible Nova-like Outburst in a Peculiar Symbiotic Binary
}

\section{Citation}

Tang, Sumin, Jonathan E. Grindlay, Maxwell Moe, Jerome A. Orosz, Robert L. Kurucz, Samuel N. Quinn, and Mathieu Servillat. 2012. “DASCH DISCOVERY OF A POSSIBLE NOVA-LIKE OUTBURST IN A PECULIAR SYMBIOTIC BINARY." The Astrophysical Journal 751 (2): 99. https:// doi.org/10.1088/0004-637x/751/2/99.

\section{Permanent link}

http://nrs.harvard.edu/urn-3:HUL.InstRepos:41399782

\section{Terms of Use}

This article was downloaded from Harvard University's DASH repository, and is made available under the terms and conditions applicable to Other Posted Material, as set forth at http:// nrs.harvard.edu/urn-3:HUL.InstRepos:dash.current.terms-of-use\#LAA

\section{Share Your Story}

The Harvard community has made this article openly available. Please share how this access benefits you. Submit a story.

Accessibility 


\title{
DASCH DISCOVERY OF A POSSIBLE NOVA-LIKE OUTBURST IN A PECULIAR SYMBIOTIC BINARY
}

\author{
Sumin Tang ${ }^{1}$, Jonathan E. Grindlay ${ }^{1}$, Maxwell Moe ${ }^{1}$, Jerome A. Orosz ${ }^{2}$, \\ Robert L. Kurucz ${ }^{1}$, Samuel N. QuinN ${ }^{1}$, and Mathieu Servillat ${ }^{1}$ \\ ${ }^{1}$ Harvard-Smithsonian Center for Astrophysics, 60 Garden St, Cambridge, MA 02138, USA; stang @cfa.harvard.edu \\ ${ }^{2}$ Astronomy Department, San Diego State University, 5500 Campanile Drive, San Diego, CA 92182-1221, USA \\ Received 2011 September 28; accepted 2012 March 23; published 2012 May 10
}

\begin{abstract}
We present photometric and spectroscopic observations of a peculiar variable (designated DASCH J075731.1+201735 or J0757) discovered from our DASCH project using the digitized Harvard College Observatory archival photographic plates. It brightened by about 1.5 mag in $B$ within a year starting in 1942, and then slowly faded back to its pre-outburst brightness from 1943 to 1950 s. The mean brightness level was stable before and after the outburst, and ellipsoidal variations with a period of $P=119.18 \pm 0.07$ days are seen, suggesting that the star is tidally distorted. Radial-velocity measurements indicate that the orbit is nearly circular $(e=0.02 \pm 0.01)$ with a spectroscopic period that is the same as the photometric period. The binary consists of a $1.1 \pm 0.3 M_{\odot}$ MOIII star, and a $0.6 \pm 0.2 M_{\odot}$ companion, very likely a white dwarf (WD). Unlike other symbiotic binaries, there is no sign of emission lines or a stellar wind in the spectra. With an outburst timescale of $\sim 10$ years and estimated $B$-band peak luminosity $M_{B} \sim 0.7, \mathrm{~J} 0757$ is different from any other known classic or symbiotic novae. The most probable explanation of the outburst is hydrogen shell burning on the WD, although an accretion-powered flare cannot be ruled out.
\end{abstract}

Key words: binaries: symbiotic - novae, cataclysmic variables

Online-only material: color figures

\section{INTRODUCTION}

Symbiotic stars are interacting binaries consisting of an evolved red giant and a hot object, which is usually a white dwarf (WD; see, e.g., Kenyon 1986, 1994; Nussbaumer 2000; Mikołajewska 2007 and references therein). Mass is transferred from the red giant to its hot companion via stellar wind in most cases, but could also be Roche lobe overflow in some cases. They usually show low temperature absorption features from the red giant, and strong emission lines from surrounding circumstellar material ionized by the hot component. Most symbiotic stars have red giant masses $0.6-3.2 M_{\odot}$, hot component masses $0.4-0.8 M_{\odot}$, and orbital periods $\sim 200-1000$ days (Mikołajewska 2003).

Symbiotic stars are highly variable sources with variation timescales from minutes to decades. The most common type of outburst, called a classical symbiotic outburst, recurs multiple times on decades-long timescales with amplitudes of 1-3 mag, and with each outburst lasts from months to a few years (Kenyon 1986; Mikołajewska 2011). The nature of these outbursts is not entirely clear and may be related to instabilities in the accretion disks (Mikołajewska 2003; Sokoloski et al. 2006). Another relatively rare type of outburst is symbiotic nova (also called slow nova), which shows a single long outburst lasting from years to decades due to thermonuclear runaways at the surface of the WD (Allen 1980; Paczyński \& Rudak 1980; Kenyon \& Webbink 1984). The peak luminosities range from 4000 to $40,000 L_{\odot}$, depending on the mass of the WD (Mürset \& Nussbaumer 1994; Iben 2003). They mimic classical novae but with much longer timescales. Only nine symbiotic novae are known so far (Belczyński et al. 2000; Mikołajewska 2011).

DASCH (Digital Access to a Sky Century @ Harvard) is a project to digitize and analyze the scientific data contained in the Harvard College Observatory photographic plates taken from the 1880s through the 1980s. The motivation is to explore the temporal variations of stars and active galaxies on the relatively poorly explored $\sim 1-100$ year timescales (but extending down to $\sim 1$ week timescales as well), as summarized by Grindlay et al. (2009) and J. Grindlay et al. (2012, in preparation). We developed the astrometry and photometry pipeline (Laycock et al. 2010; Los et al. 2011; Servillat et al. 2011; S. Tang et al., in preparation), and have scanned $\sim 19,000$ plates in five initial fields. Here we report the discovery of an unusual 10 year outburst in a peculiar symbiotic system, DASCH J075731.1+201735 (named after its equatorial J2000 coordinate, hereafter J0757).

\section{OBSERVATIONS AND RESULTS}

\subsection{DASCH and ASAS Light Curves}

J0757 was measured on 694 plates near the M44 field noted by its peculiar long-term variability. These plates cover $5^{\circ}-25^{\circ}$ on a side with typical limiting magnitudes $B \sim 14-15 \mathrm{mag}$, and most of them are blue sensitive emulsions. We used the GSC 2.3.2 catalog (Lasker et al. 1990) for photometric calibration, and our typical uncertainty is $\sim 0.1-0.15 \mathrm{mag}$ (Laycock et al. 2010; S. Tang et al., in preparation). There are $\sim 1.2 \times 10^{5}$ objects with more than 100 mag measurements distributed over $\sim 100$ years from these plates covering the M44 field. J0757, which is N2211021132 in the GSC catalog, is the only one found with $>1$ mag outburst on a $\sim 10$ year timescale above a well-defined quiescence level.

The DASCH light curve of J0757 is shown in Figure 1. We supplement the figure with $V$-band data from ASAS starting in the year 2000 (J0757 has the designation ASAS J075731+2017.6; Pojmanski 2002). J0757 is classified by ASAS as a semi-detached/contact binary with best-fit period of 119.2 days with $0.16 \mathrm{mag}$ variations in the $V$ band. Since ASAS data are in the $V$ band, while DASCH magnitudes are in $B$, we added 1.5 mag to the ASAS $V$ mag in the plot, which is about 

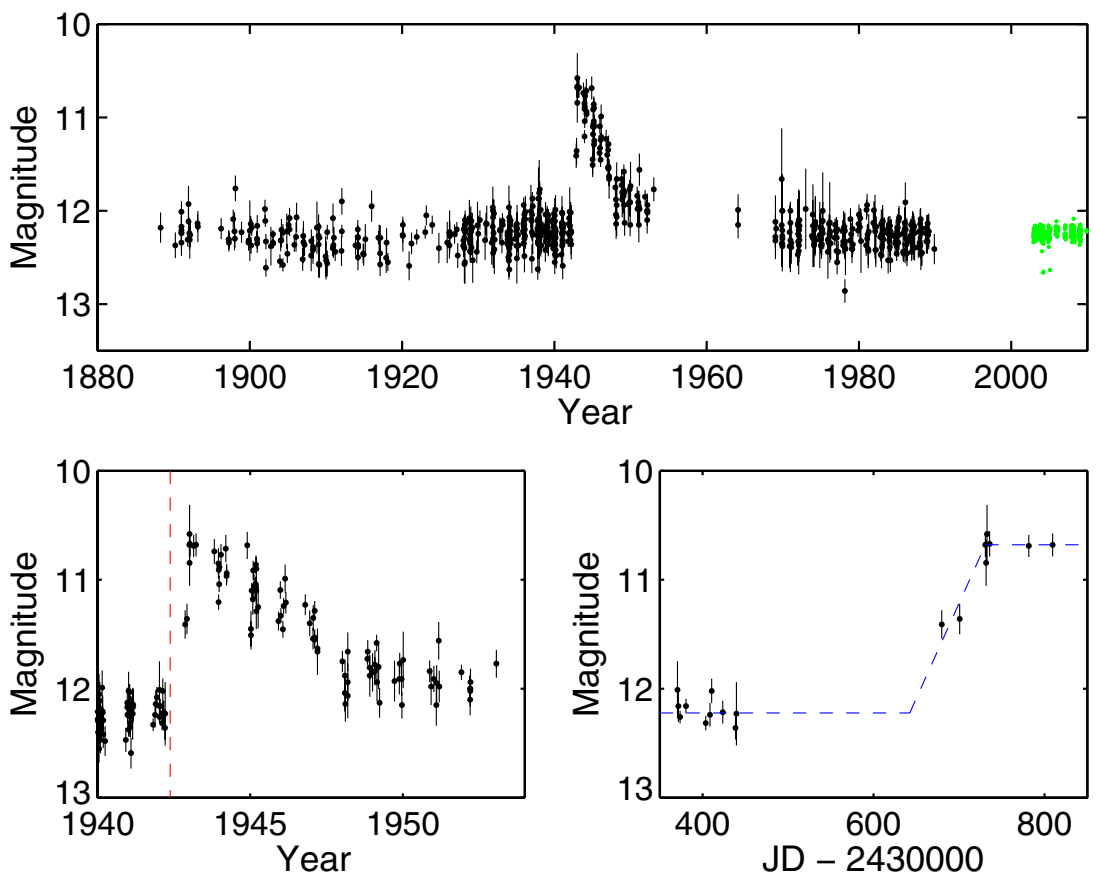

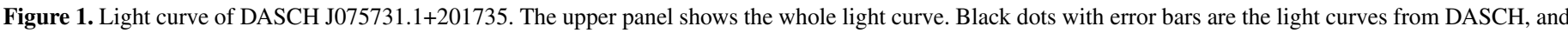

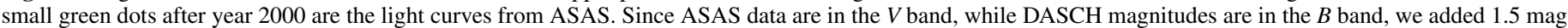

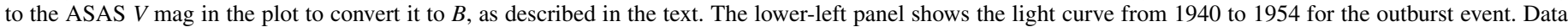

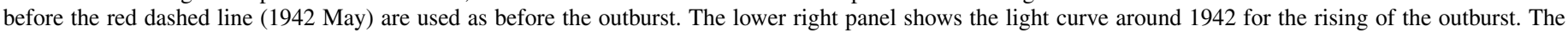

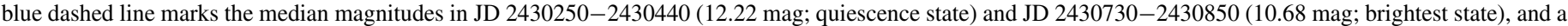
naive linear increase in between. The time span of the linear increase is 88.5 days.

(A color version of this figure is available in the online journal.)

the sum of the maximum galactic extinction $(E(B-V)=0.06$; Schlegel et al. 1998) and the typical $B-V$ value for an M0III star (1.43; Pickles 1998). We will discuss the spectral classification in Section 2.2.

As shown in Figure 1, J0757 was in quiescence until about 1942 March. J0757 then brightened over the rest of the year of 1942, and slowly decayed until about 1950 . The peak magnitude was reached around 1943 January. There is a seasonal gap between 1942 April and October when the star was low or not visible in the night sky, and thus we do not know when exactly the outburst started. If we naively assume that its magnitude increased linearly, then the outburst rise time is $\sim 88.5$ days (see the lower right panel in Figure 1).

Before and after the outburst when data are available (i.e., 1890-1942 and 1962-1990), the magnitudes of J0757 were reasonably stable (note that the relatively constant $B \sim 11.9$ from $\sim 1950-1954$ was not yet the quiescent value). The median DASCH magnitude before the outburst (e.g., before 1942 June) is 12.24 and the median magnitude after the outburst (after 1960 ) is 12.26. The median magnitude of the entire DASCH light curve excluding the period between 1942 June and the start of 1960 is 12.25 , with rms scatter $=0.14$ mag for the 607 data points outside the outburst. The median ASAS $V$ magnitude is $10.75 \mathrm{mag}$, which corresponds to $B=12.24$, assuming the typical colors of an MOIII star with galactic extinction.

\subsection{Light Curve Folding and the Photometric Period}

Both the DASCH and ASAS light curves show ellipsoidal variations. The ellipsoidal variation amplitude is $\sim 0.11 \mathrm{mag}$ in the DASCH data ( $B$ band, 1890-1942 and 1962-1990), and $\sim 0.16$ mag in the ASAS data ( $V$ band, 2003-2009).
We folded the light curves of J0757 to search for its photometric period. We divided DASCH light curve into two phases, i.e., before the outburst and after the outburst, as described in Section 2.1. We then got four sets of light curve data: ASAS light curve, DASCH light curve excluding the outburst (before or after the outburst), DASCH light curve before the outburst, and DASCH light curve after the outburst.

We first folded the ASAS light curve with a given period, and fit it with a three harmonics Fourier model (an example is shown as the blue line in the upper panel of Figure 2). We fixed the model line in shape, but allow two free parameters, i.e., median magnitude and amplitude, for adjustments. Next, we fit the four sets of light curve data to the three harmonics Fourier model, and derived the reduced $\chi^{2}$ as a function of different trial periods, as shown in the lower panels in Figure 2. DASCH data were binned by phase at the given period in the fitting. Our best-fit period is $119.18 \pm 0.07$ days. The fact that the binary period is unchanged (within the errors) sets limits on the change of the orbit (due to mass transfer or onset of common envelope evolution). The resulting value for $\dot{a} / a$ is $(0 \pm 4) \times 10^{-6} \mathrm{yr}^{-1}$.

\subsection{Optical Spectroscopy}

Spectra were obtained with the FAST spectrograph (Fabricant et al. 1998) on the $1.5 \mathrm{~m}$ Tillinghast reflector telescope at the F. L. Whipple Observatory (FLWO) for spectral classification. The spectra were reduced and wavelength calibrated with standard packages (Tokarz \& Roll 1997). Figure 3 shows the FAST 300 grating spectrum, which has a resolution of about $7 \AA$. The upper panel shows the whole spectrum, which suggests that its spectral type is M0. The lower panels show two luminosity indicators, which suggest that DASCH J0757 belongs to luminosity class III. The lower-left panel shows 

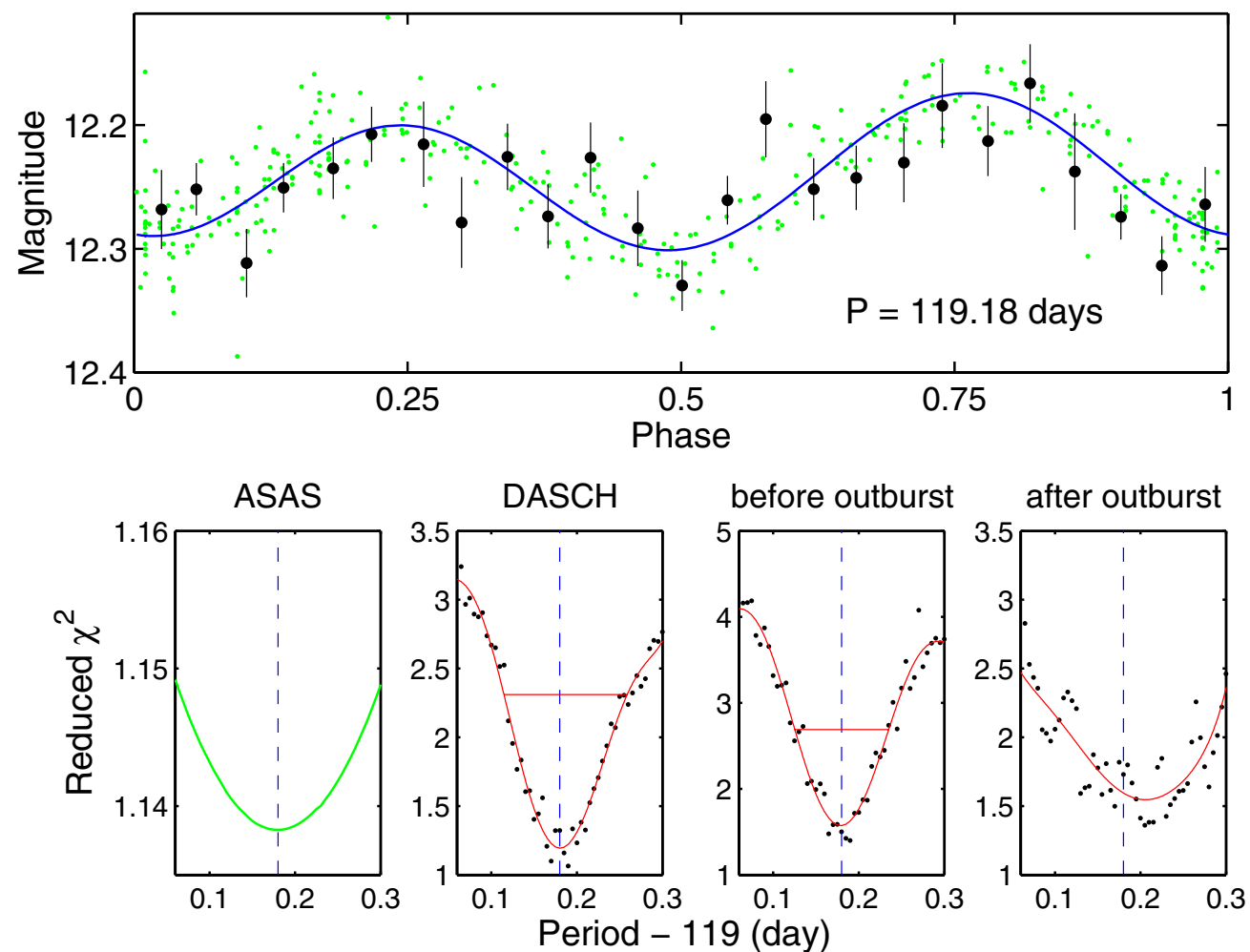

Figure 2. Folded light curve and period search of DASCH J0757. The upper panel shows the folded light curve with $P=119.18$ days and $T_{0}=2455597.98$. Black solid dots are DASCH data binned by phase, with error bars representing the standard deviation of the mean. Small green dots are ASAS data. Blue solid line is the best-fit three harmonics Fourier model derived from the ASAS data. The lower panels show the reduced $\chi^{2}$ as a function of folding period. From left to right are the reduced $\chi^{2}$ using ASAS data, DASCH data excluding the outburst, DASCH data before the outburst (before 1942 June, as indicated as the red dashed line in the lower-left panel in Figure 1), and DASCH data after the outburst (after 1960). The black dots are the reduced $\chi$-square values, and the red lines are a sixth-order polynomial fit. In the two lower middle panels, i.e., DASCH data excluding the outburst, and DASCH data before the outburst, $1 \sigma$ confidence levels by assuming $\chi^{2}$ distribution are marked as red horizontal lines. The more limited DASCH data after the outburst may suggest a $\sim 0.02$ days longer period, but this is not a significant deviation. The vertical blue dashed lines mark the best-fit period of 119.18 days.

(A color version of this figure is available in the online journal.)
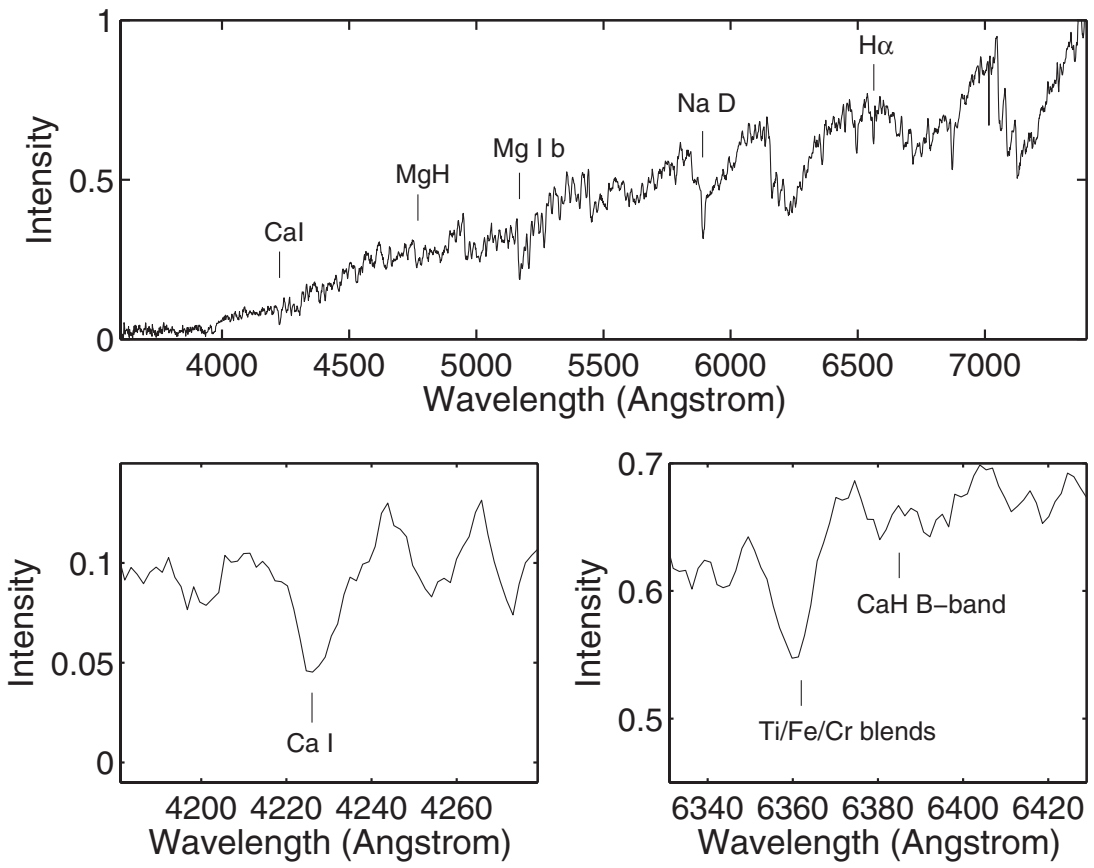

Figure 3. FAST 300 grating spectrum of DASCH J0757. Spectral resolution is about $7 \AA$. The upper panel shows the whole spectrum. The lower panels show two luminosity indicators, $\mathrm{Ca}$ I at $4226 \AA$, and the $\mathrm{CaH} B$ band at $6385 \AA$ with the blend near $6362 \AA$ consisting of Ti, $\mathrm{Fe}$, and $\mathrm{Cr}$ on the left. 

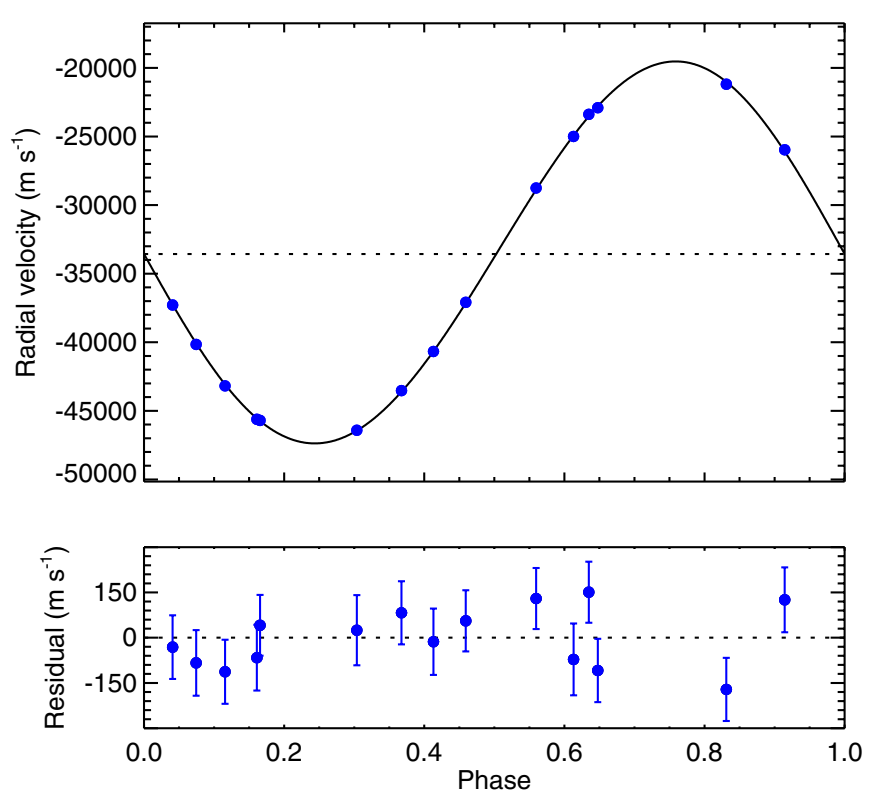

Figure 4. Radial velocity of DASCH J0757 as a function of orbital phase, using the ephemeris in Table 1. Blue solid dots are observation data using the TRES spectrograph at the FLWO $1.5 \mathrm{~m}$, and the smooth curve is the best-fitting model. Residuals from the fit are given in the lower panel.

(A color version of this figure is available in the online journal.)

Ca I at $4226 \AA$, which disappears with increasing luminosity, indicating that DASCH J0757 is not a luminosity class I star. The lower-right panel shows the $\mathrm{CaH} B$ band at $6385 \AA$, and the blend near $6362 \AA$ consisting of Ti, Fe, and Cr. The weakness of $\mathrm{CaH} B$ band suggests that DASCH J0757 is not a main-sequence (MS) star. We therefore adopt a spectral type of $\sim$ MOIII for DASCH J0757, with uncertainties of $\sim \pm 1$ in both spectral type and luminosity class.

We also obtained spectra using the MIKE echelle spectrograph (Bernstein et al. 2003) on the $6.5 \mathrm{~m}$ Magallen/Clay telescope with a $0.7 \times 5^{\prime \prime}$ slit on 2011 January 17 . The spectral resolution is $R \sim 30,000$. The spectra were extracted using an IDL pipeline developed by S. Burles, R. Bernstein, and J. S. Prochaska. ${ }^{3}$ The reduced spectra have signal-to-noise ratio $(\mathrm{S} / \mathrm{N}) \sim 50-100$ in $4300-9000 \AA$ and $\mathrm{S} / \mathrm{N} \gtrsim 10$ in $3700-4300 \AA$. We computed a grid of spectrum templates for a range of abundances, effective temperatures, and gravities using Kurucz's programs ATLAS12 and SYNTHE (Kurucz 2005). We derived the projected rotation velocity of $10 \pm 1 \mathrm{~km} \mathrm{~s}^{-1}$. In $\mathrm{M}$ giants the continuum opacity is mainly $\mathrm{H}$ - which varies with metal abundance. The ratio of line to continuum does not change strongly with abundance. The TiO background is strong at any abundance because both $\mathrm{Ti}$ and $\mathrm{O}$ are alpha enhanced at low abundances. We estimated an effective temperature of $T_{\text {eff }}=3850 \pm 50 \mathrm{~K} ;$ a surface gravity of $\log g=1.0 \pm 0.5 \mathrm{cgs}$; and a metallicity of $[\mathrm{Fe} / \mathrm{H}]=-0.6 \pm 0.1$, assuming $\mathrm{J} 0757$ is a population II star with alpha enhancements. The spectra can also be fit with Population I solar abundance templates. There is no sign of emission lines or wind in its spectra.

To measure the radial-velocity curve of J0757, we obtained spectra on 15 different nights from 2010 October through 2011 April with the Tillinghast Reflector Echelle Spectrograph (TRES; Szentgyorgyi \& Furész 2007) on FLWO $1.5 \mathrm{~m}$. These spectra were reduced and wavelength calibrated with standard packages (Mink 2011). The spectral resolution is $R \sim 44,000$.

\footnotetext{
3 http://web.mit.edu/ burles/www/MIKE/
}

Table 1

Astrophysical Parameters for DASCH J0757

\begin{tabular}{|c|c|}
\hline Parameter & Value \\
\hline \multicolumn{2}{|c|}{ Observational parameters with highest confidence } \\
\hline Spectral type & M0III \\
\hline$T_{0}(\mathrm{HJD})$ & $2455597.980 \pm 0.054$ \\
\hline Period (days) & $119.18 \pm 0.07$ \\
\hline$\gamma\left(\mathrm{km} \mathrm{s}^{-1}\right)$ & $-33.57 \pm 0.04$ \\
\hline$K_{1}\left(\mathrm{~km} \mathrm{~s}^{-1}\right)$ & $13.906 \pm 0.025$ \\
\hline Eccentricity & $0.025 \pm 0.01$ \\
\hline$\omega(\operatorname{deg})$ & $70.49 \pm 0.09$ \\
\hline$V_{\text {rot }} \sin i\left(\mathrm{~km} \mathrm{~s}^{-1}\right)$ & $10 \pm 1$ \\
\hline \multicolumn{2}{|c|}{ Parameters derived from atmosphere fitting } \\
\hline$T_{\text {eff }}(\mathrm{K})$ & $3850 \pm 50$ \\
\hline $\log g(\mathrm{cgs})$ & $1.0 \pm 0.5$ \\
\hline$[\mathrm{Fe} / \mathrm{H}]$ & $-0.6 \pm 0.1$ \\
\hline \multicolumn{2}{|l|}{ Estimates based on models } \\
\hline$i(\mathrm{deg})$ & $60 \pm 15$ \\
\hline$M_{1}\left(M_{\odot}\right)$ & $1.1 \pm 0.3$ \\
\hline$M_{2}\left(M_{\odot}\right)$ & $0.6 \pm 0.2$ \\
\hline Orbital separation $\left(R_{\odot}\right)$ & $120 \pm 11$ \\
\hline$R_{1}\left(R_{\odot}\right)$ & $35 \pm 9$ \\
\hline$L_{1}\left(L_{\odot}\right)$ & $250 \pm 130$ \\
\hline Distance (kpc) & $1.0 \pm 0.3$ \\
\hline$h_{z}(\mathrm{pc})$ & $400 \pm 110$ \\
\hline$M_{B}$ in quiescence & $2.2 \pm 0.6$ \\
\hline$M_{B}$ outburst & $0.7 \pm 0.6$ \\
\hline$L_{\text {outburst peak }}\left(L_{\odot}\right)$ & $\begin{array}{c}10^{2}-10^{4} \text { (if no constraint on } T_{\text {hot }} \text { ) } \\
\left.100 \text { (if } T_{\text {hot }} \sim 8100 \mathrm{~K}\right) \\
2.5 \times 10^{4}\left(\text { if } T_{\text {hot }} \sim 90,000 \mathrm{~K}\right)\end{array}$ \\
\hline$L_{\text {hot }}$ in quiescence $\left(L_{\odot}\right)$ & $\sim 2-8$ \\
\hline$\dot{M}$ in quiescence $\left(M_{\odot} \mathrm{yr}^{-1}\right)$ & $\sim(1-4) \times 10^{-9}$ \\
\hline
\end{tabular}

Note. Details are discussed in Sections 2 and 3.

The reduced spectra have typical S/N 10-30 in 4500-9000 , and $\mathrm{S} / \mathrm{N} \sim 5-10$ in $4000-4500 \AA$.

Radial velocities of DASCH J0757 were derived from the TRES spectra and were fitted to a model velocity curve using the derived photometric period (see Figure 4). The derived orbital parameters are listed in Table 1. The resulting mass function is

$$
f_{1}\left(M_{2}\right)=(3.31 \pm 0.02) \times 10^{-2} M_{\odot},
$$

and

$$
M_{2} \sin i=(0.3211 \pm 0.0006)\left(M_{1}+M_{2}\right)^{2 / 3} M_{\odot},
$$

where subscript 1 denotes the red giant and 2 denotes its companion.

For comparison, we have also fit a model velocity curve without using the photometric period of $P=119.18 \pm$ 0.07 days, and got similar results: $T_{0}=2455597.969 \pm 0.06$, $P=118.89 \pm 0.080$ days, $\gamma=-33.57 \pm 0.04 \mathrm{~km} \mathrm{~s}^{-1}$, $K=13.885 \pm 0.023 \mathrm{~km} \mathrm{~s}^{-1}, e=0.024 \pm 0.015$, and $\omega=70.38 \pm 0.09$. In the following discussion throughout the paper, we adopt the parameters obtained using the photometric period of $P=119.18 \pm 0.07$ days, as listed in Table 1 .

\subsection{SED from Archival Data}

Using archival data from WISE, IRAS, AKARI/IRAC, 2MASS, FON, SDSS, GALEX, and ROSAT, we plotted the spectral energy distribution (SED) of J0757, as shown in Figure 5. 


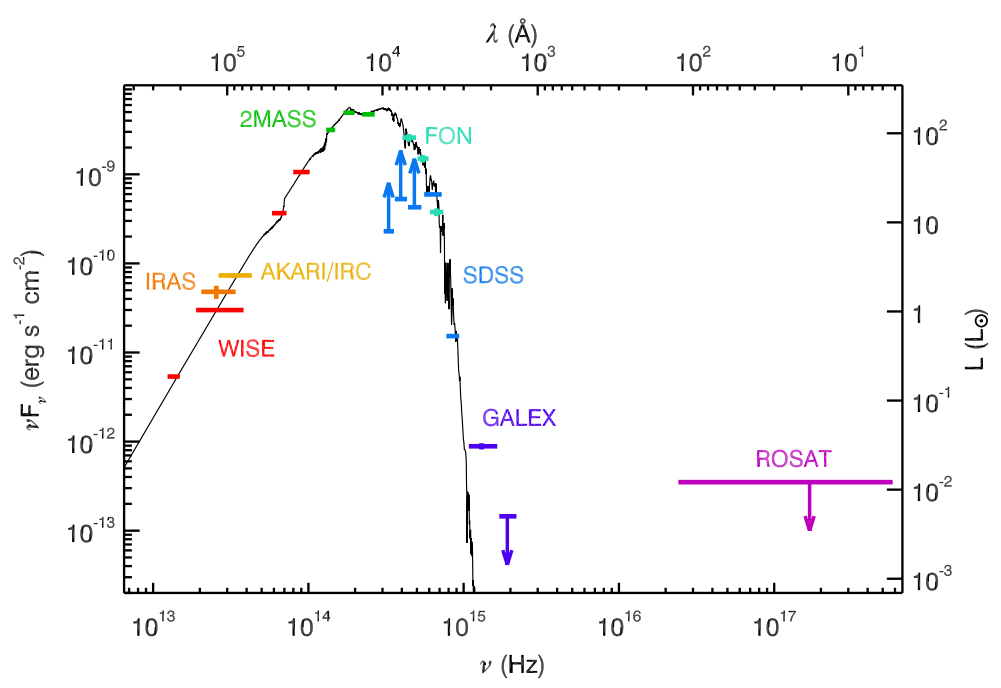

Figure 5. SED of DASCH J0757 using archival data. The black line represents a synthetic spectrum of a $T_{\text {eff }}=3750 \mathrm{~K}$, and $\log g=1.5$ giant (Lejeune et al. 1997 ). Note that SDSS riz bands are saturated. It was not detected by GALEX FUV and ROSAT.

(A color version of this figure is available in the online journal.)

The phase of the GALEX observation is $0.603 \pm 0.01$, and the phase of ROSAT observation is $0.426 \pm 0.02$. Here phase 0.5 is defined as inferior conjunction of the red giant. The SED is well matched by a synthetic spectrum of a $T_{\text {eff }}=3750 \mathrm{~K}$, and $\log g=1.5$ giant (Lejeune et al. 1997), from IR to optical, as shown in the black line in Figure 6. However, there is an excess in the GALEX NUV ( 1900-2700 $)$, which suggests a hot component in addition to the giant.

\section{SYSTEM PARAMETERS}

\subsection{Ellipsoidal Models}

We derive system parameters from the light and velocity curves. For this purpose we used the ELC code of Orosz \& Hauschildt (2000) to model the ASAS $V$-band light curve and the TRES radial-velocity curve. The ELC model is based on standard Roche geometry and uses specific intensities from model atmospheres, in particular the NEXTGEN models of Hauschildt et al. (1999). ELC's use of the model atmosphere specific intensities eliminates the need for a parameterized limb darkening law, which is important in cases with red giants where the limb darkening near the stellar limb is very nonlinear (Orosz \& Hauschildt 2000). We do not have model atmosphere specific intensities appropriate for WDs. However, since the red giant dominates the $V$-band flux, this is not a serious limitation.

In our initial runs, we had eight free parameters: the orbital period $P$, the time of periastron passage $T_{0}$, the inclination $i$, the Roche lobe filling factor $f_{1}$, the eccentricity $e$, the argument of periastron $\omega$, the $K$ velocity of the red giant $K$, and the mass of the red giant $M_{1}$. We assumed synchronous rotation. The models are somewhat insensitive to the temperature of the red giant, and that parameter was held fixed at $T_{1}=3720 \mathrm{~K}$. In addition to the light and velocity curve, we used the projected rotational velocity of the red giant as an extra constraint.

The model fits were optimized using ELC's genetic code and its Monte Carlo Markov Chain code. After inspection of the post-fit residuals, additional free parameters were considered. We allowed the red giant's rotation to be asynchronous with the orbit, where $\Omega$ is the ratio of the spin frequency to the orbital frequency. We also accounted for heating by the WD. In this case, we fixed the luminosity of the irradiating source to
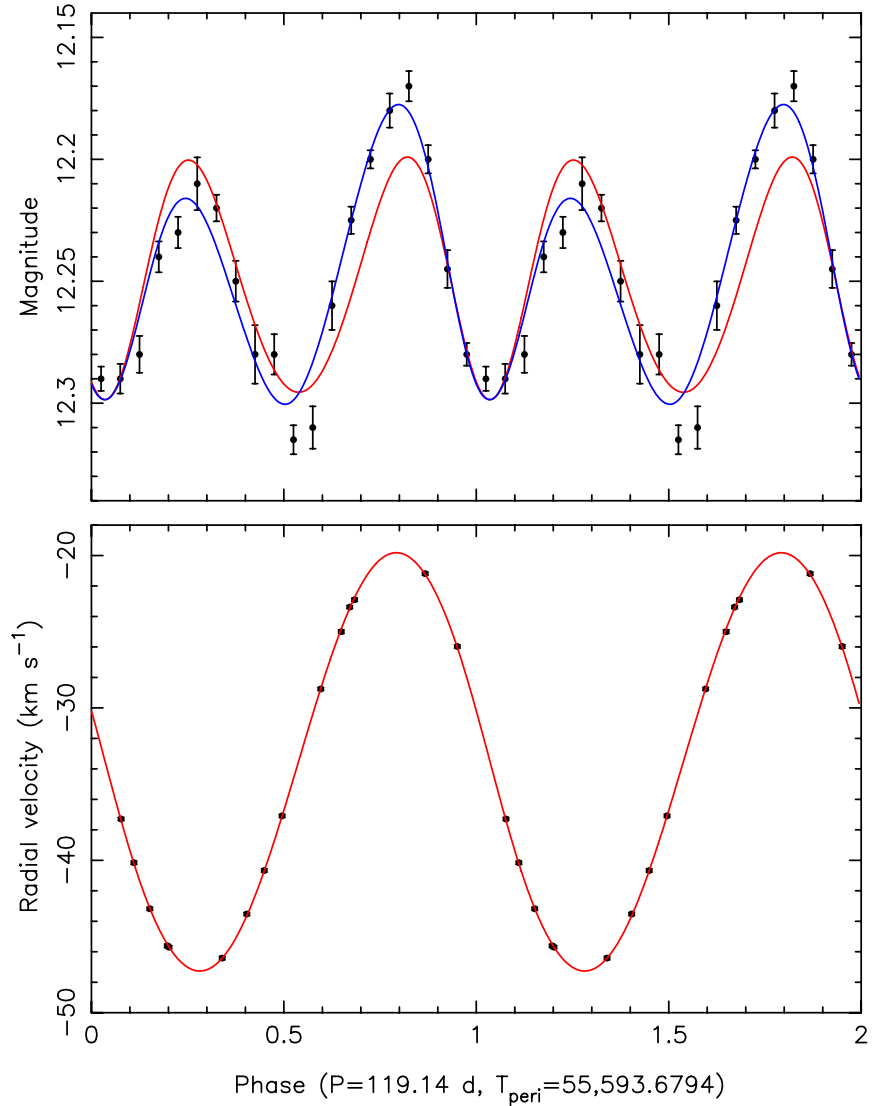

Figure 6. Top: the phased and binned ASAS $V$-band light curve of J7057 and some representative ellipsoidal models. The red line is a model that includes irradiation and non synchronous rotation, and the blue line is a model that has an accretion disk with a hot spot. Bottom: the TRES radial-velocity measurements phased on the same ephemeris (which uses the time of periastron passage as the zero point) and the best-fitting model.

(A color version of this figure is available in the online journal.)

$\log L_{i}=36.6$ (cgs units) and allowed the albedo $A_{1}$ to be a free parameter (the choice of $A_{1}$ is somewhat arbitrary since different combinations of $L_{i}$ and $A_{1}$ can give identical light curves). Finally, we included an accretion disk that is parameterized by 
the outer radius in terms of the Roche radius $r_{\text {out }}$, the temperature at the inner $\operatorname{rim} T_{d}$, and the exponent of the disk temperature distribution $\xi$, where $T(r)=T_{d}\left(r / r_{i}\right)^{\xi}$.

Some typical results are shown in Figure 6. The binned ASAS light curve has maxima with different heights, which could be indicative of an eccentric orbit. However, the simultaneous fit to the radial velocities indicates a small eccentricity (0.012). Also, the minimum near phase 0.5 , corresponding to the inferior conjunction of the red giant, is deeper than the minimum at phase 0.0 . For a pure ellipsoidal light curve, the minimum at phase 0.0 would be the deeper one. Heating by the irradiating source "fills in" the minimum near phase 0 . However, in all of the models considered, we never had a case where the model curve had a deeper minimum near phase 0.5 , so simple heating cannot explain the difference in the observed minima.

An accretion disk could potentially eclipse the red giant near phase 0 , thereby making the minimum there deeper. This is opposite of what we want. Normally, the accretion disk does not give phase modulated light in the ELC model (if it is not eclipsed by the star). However, a "hot spot" on the disk rim can be used to mimic the place where the matter transfer stream impacts the disk. As the binary turns in space, different areas of this spot are visible, giving rise to extra light at certain phases. An example model is shown in Figure 6. The hot spot has an angular width of $18^{\circ}$, an azimuth (measured from the line of centers in the direction or the orbital motion) of $66^{\circ}$, and has a temperature of $3800 \mathrm{~K}$, which is 4.3 times hotter than the rest of the disk rim. The maximum near phase 0.75 is fit, but the minimum near phase 0.5 is still not well fit.

We did two runs of fitting with a Monte Carlo Markov Chain, one with an external constraint on the rotational velocity and one without the constraint. Results from both runs are similar. The best-fit ellipsoidal models have inclination of $60^{\circ} \pm 5^{\circ}$, WD mass of $0.5 \pm 0.1 M_{\odot}$, red giant mass of $1 \pm 0.3 M_{\odot}$, and red giant radius of $43 \pm 4 R_{\odot}$.

We note that in order to match the observed rotational velocity of $V_{\text {rot }} \sin i=10 \pm 1 \mathrm{~km} \mathrm{~s}^{-1}$ derived from the MIKE spectra, the red giant must be rotating 0.7 times slower than its synchronous velocity. Given the typical tidal synchronization timescale of $\sim 10^{5}$ yr (Zahn 1989) adopting the parameters listed in Table 1, the red giant is expected to be corotating with the binary. Consequently, the size of the giant should be $R_{1}=P V_{\text {rot }} / 2 \pi=23.5 / \sin i R_{\odot}$, which is $27 R_{\odot}$ if $i=60^{\circ}$, which are smaller than the radius derived from ellipsoidal fitting. Such a discrepancy has been seen in many symbiotic binaries and is an unresolved "embarrassing problem" (Mikołajewska 2007). It probably comes from the extended atmosphere, and that limb darkening near the limb is very nonlinear. The light curve models with near-linear limb darkening produce ellipsoidal light curves with amplitudes that are too small. Also, the rotational broadening kernel can be a lot different than the usual analytic kernel, which in turn leads to strange biases in the $V_{\text {rot }} \sin i$ value (Orosz \& Hauschildt 2000).

\subsection{Estimates from the Spectroscopic Observations}

Here we estimate the astrophysical parameters of DASCH J0757 based on the results in Sections 2 and 3.1. Since the galactic extinction is small $(E(B-V)=0.06$; Schlegel et al. 1998), we do not take it into account for simplicity. The following estimated parameters are summarized in Table 1.

The inclination from the ellipsoidal fitting is $i \sim 55^{\circ}-65^{\circ}$, and we adopt a more tolerant value of $i=45^{\circ}-75^{\circ}$ in the following estimates. The radius of the red giant ranges from $\sim 25-33 R_{\odot}$ (from $V_{\text {rot }}$ assuming synchronized rotation) to $\sim 43 R_{\odot}$ (from ellipsoidal fitting), as discussed in Section 3.1. Therefore, we adopt a radius and error bar that encompasses both methods as $R_{1}=35 \pm 9 R_{\odot}$. At $T_{\text {eff }}=3850 \pm 50 \mathrm{~K}$, the bolometric luminosity of the red giant is $L=250 \pm 130 L_{\odot}$, and its distance is $1.0 \pm 0.3 \mathrm{kpc}$. The absolute magnitude of the system in quiescence is then $M_{B}=2.2 \pm 0.6$.

Combining the radius, effective temperature, $\log g=1.0 \pm$ $0.5 \mathrm{cgs}$, and $[\mathrm{Fe} / \mathrm{H}]=-0.6 \pm 0.1$, the red giant mass inferred from stellar evolutionary models (Bertelli et al. 2008) is $M_{1} \sim 0.9 \pm 0.4 M_{\odot}$. This mass estimate for the giant is determined solely from the equations of stellar structure and is therefore independent of its prior evolution. The mass of the giant may have been greater in the past. However, considering the giant is only partially filling its Roche lobe as inferred from the ellipsoidal fitting, and given the M0III wind loss rate of $\sim 10^{-9}-10^{-8} M_{\odot} \mathrm{yr}^{-1}$ (Cox 2000), we do not expect it has lost a significant fraction of its current mass over the $\sim 10^{7} \mathrm{yr}$ lifetime of the giant in its current phase of evolution. Requiring $0.8 M_{\odot}$ to turnoff from MS within a Hubble time, we then have $M_{1} \sim 1.1 \pm 0.3 M_{\odot}$. From Equation (2) and our adopted $i=$ $45^{\circ}-75^{\circ}$, this implies the secondary mass is $M_{2} \sim 0.6 \pm 0.2 M_{\odot}$, and the orbital separation $a \sim 120 \pm 11 R_{\odot}$. Therefore, the Roche lobe radii of the two stars are $R_{\mathrm{RL}, 1} \sim 53 \pm 4 R_{\odot}$ and $R_{\mathrm{RL}, 2} \sim 38 \pm 6 R_{\odot}$ for the $\mathrm{M}$ giant and the secondary, respectively (Eggleton 1983). The Roche lobe filling factor of the $\mathrm{M}$ giant is then $f_{\mathrm{RL}}=R_{1} / R_{\mathrm{RL}, 1}=0.66 \pm 0.17$.

If the deviation of the ASAS folded light curve and the ellipsoidal model with a giant alone is caused by a hot component due to a WD companion, its accretion disk and/or a tidal stream, then it is about $3 \%$ the luminosity of the giant in the $V$ band. The luminosity of the hot component is then $2-8 L_{\odot}$, for an effective temperature in the range of 3800-10,000 K. For an $M_{2} \sim 0.6 M_{\odot}$ WD with $R_{2} \sim 0.01 R_{\odot}$, assuming all the gravitational potential energy is converted to thermal radiation, the corresponding accretion rate is $(1-4) \times 10^{-9} M_{\odot} \mathrm{yr}^{-1}$.

During the 1942-1950s outburst, the $B$ magnitude of J0757 increased by $\sim 1.5 \mathrm{mag}$ at the peak. At a distance of $D \sim 1 \mathrm{kpc}$, the absolute $B$ magnitude of the outburst is $M_{B \text {, outburst peak }} \sim$ 0.7 mag. The peak bolometric luminosity of the outburst

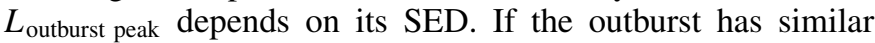
SED as an MOIII star, then $L_{\text {outburst peak }} \sim 750 L_{\odot}$. The minimum required bolometric luminosity would be when the radiation is peaked in the $B$ band, i.e., $L_{\text {outburst peak }} \sim 100 L_{\odot}$ at $T \sim 8100 \mathrm{~K}$. Such a temperature is consistent with the observed hot component temperature of CH Cyg during its 1992 outburst powered by accretion (Skopal et al. 1996). If the outburst is powered by hydrogen shell burning, the temperature during the outburst would be much hotter, i.e., $T_{\text {outburst }} \sim 10^{5} \mathrm{~K}$ as observed in some symbiotic stars (Mürset et al. 1991). For example, Z And is found to have a temperature of $\sim 9 \times 10^{4} \mathrm{~K}$ during its 2000-2002 outburst (Sokoloski et al. 2006). If the outburst in $\mathrm{J} 0757$ has a temperature of $9 \times 10^{4} \mathrm{~K}$, most of the radiation is in the UV, and the corresponding bolometric luminosity of $\Delta B=1.5$ mag increase is $L_{\text {outburst peak }} \sim 2.5 \times 10^{4} L_{\odot}$ assuming black body radiation.

\section{DISCUSSION}

The amplitude and timescale of the 1940s outburst, and its stability during quiescence phases (except the periodic ellipsoidal variation), is very unusual. The mass loss rate of an MOIII star is $\sim 10^{-9}-10^{-8} M_{\odot} \mathrm{yr}^{-1}$ (Cox 2000). With a Roche lobe filling factor $\sim 0.5-0.8$ as discussed in Section 3.2, 
a significant fraction of the wind could be transferred to its companion via wind Roche lobe overflow (Podsiadlowski \& Mohamed 2007). If the secondary is a $0.6 M_{\odot}$ MS star with $R \sim 0.6 R_{\odot}$, the only way to power the outburst is by gravitational energy release. The least required accretion rate to power the 1940 s outburst of $\mathrm{J} 0757$ is then $3 \times 10^{-6} M_{\odot} \mathrm{yr}^{-1}$ $\left(L_{\text {peak }} \sim 100 L_{\odot}\right.$ if $T \sim 8100 \mathrm{~K}$ ), which is $2-3$ orders of magnitude higher compared with the typical mass loss rate of the $\mathrm{M}$ giant. Therefore, a compact object, almost certainly a WD (at $\sim 0.6 M_{\odot}$, it cannot be a neutron star or a black hole), is needed to power the outburst.

If the outburst was powered entirely by accretion on to a $0.6 M_{\odot} \mathrm{WD}$, the least required accretion rate to power the 1940s outburst of $\mathrm{J} 0757$ is $5 \times 10^{-8} M_{\odot} \mathrm{yr}^{-1}$, assuming all the potential energy is converted into radiation $\left(L_{\text {peak }} \sim 100 L_{\odot}\right.$ if $T \sim 8100 \mathrm{~K})$. However, J0757 only showed one single outburst and was stable before and after the outburst, which is different from other symbiotic systems with accretion-powered outbursts, such as PU Vul (before its nuclear runaway outburst in 1977), which was highly variable with multi-epoch outbursts (Liller \& Liller 1979). Another symbiotic variable powered by accretion, CH Cyg, was stable prior to 1963 for several decades, but later showed a series of outbursts since the onset of activity in 1963 (Mikołajewski et al. 1990).

The most probable explanation of the J0757 outburst is then hydrogen burning on the surface of the WD. The outburst profile of J0757, i.e., a relatively sharp rise and exponential-like decay, closely resembles that of Z And and CI Cyg, which are believed to be related to the presence of unstable accretion disk around a WD with hydrogen burning shell (Mikołajewska 2003; Mikołajewska et al. 2002). A "combination nova" outburst on $\mathrm{Z}$ And has been reported by Sokoloski et al. (2006), which is triggered by an accretion instability and resulted in increased thermonuclear burning. The outburst of J0757 may have started at the stable burning zone $\left(\dot{M}>\dot{M}_{\text {crit }}=3.3 \times 10^{-8} M_{\odot} \mathrm{yr}^{-1}\right.$ for a $0.6 M_{\odot}$ WD; Nomoto 1982; Nomoto et al. 2007; Shen \& Bildsten 2007) with an increased accretion rate due to an accretion disk instability (King et al. 2003), when the accumulated envelope mass on the WD exceeded the critical value (3× $10^{-5} M_{\odot}$; Townsley \& Bildsten 2004). Then, consequently, hydrogen was ignited and started quasi-steady burning to power the outburst. The luminosity of such a quasi-steady burning increases with higher WD mass, as well as higher accretion rate. For a $0.6 M_{\odot} \mathrm{WD}$, the theoretical luminosity at $\dot{M}_{\text {crit }}$ is $L \sim 10^{3.4} L_{\odot}$, and the effective temperature is $\sim 2.5 \times 10^{5} \mathrm{~K}$. If $\dot{M} \sim 4 \dot{M}_{\text {crit }}$, then the luminosity is $\sim 10^{4} L_{\odot}$, and the effective temperature is $\sim 8 \times 10^{4} \mathrm{~K}$ (Nomoto et al. 2007). Such a range of luminosity and corresponding temperature is consistent with the estimated peak luminosity of J0757, which is $\sim 10^{4} L_{\odot}$ if $T \sim 10^{5} \mathrm{~K}$.

The timescale of the hydrogen shell burning strongly depends on the mass of the WD, accretion rate, and whether a optically thick wind occurs (Kato \& Hachisu 1994; Prialnik \& Kovetz 1995). Kato et al. (2011) suggested that the companion star plays an important role in producing a wind, and thus affects the timescale of the outburst. As shown in Figure 6 in Kato et al. (2011), in wide symbiotic binaries like PU Vul $\left(P_{\text {orb }}=\right.$ 4900 days), the expanded WD envelope is not affected by the companion, resulting in a quiet evolution without strong winds in the outburst, and thus longer outburst (peak plateau timescale of $\sim 10$ years). On the other hand, in close binaries such as V723 Cas and HR Del ( $P_{\text {orb }}<1$ day) with similar WD masses as PU Vul $\left(\sim 0.6 M_{\odot}\right)$, the companion would have been deeply embedded in the nova envelope, resulted in strong winds and much shorter outburst (peak plateau timescale of $\sim 200$ days). We note that $\mathrm{J} 0757$, with $P_{\text {orb }}=119.2$ days and a peak plateau lifetime of $\sim 1$ years (as shown in the lower-left panel in Figure 1), lies interestingly in the region between the canonical symbiotic novae such as PU Vul, and novae in close binaries such as V723 Cas.

The absence of emission lines in the spectra of $\mathrm{J} 0757$ is peculiar, but not incompatible with the hydrogen burning powered outburst picture. Nova emission lines disappear quite rapidly after the outburst (Downes et al. 2001), and it is not surprising that we do not see emission lines 60 years after the outburst. The lack of emission lines could be due to either or both of the following. The first possible reason is that there is no significant surrounding material. This may be either due to the small amount of mass loss in the system even during the nova outburst, which is consistent with the recent results by Starrfield et al. (2012), or due to the fact that ejected shells disappear very fast, such as observed in V723 Cas (Krautter et al. 2002). The second possible reason is the lack of ionizing UV photons. A post-nova WD can cool off fast, for example, the WD in DQ Her has been found to be below $10 L_{\odot} 40$ years after the nova outburst (Stasiǹska \& Tylenda 1990). As discussed in Sections 2 and 3 , the luminosity of the hot component in J0757 is only a few $L_{\odot}$, while the luminosity of the M giant is $\sim 250 L_{\odot}$, which dominates the spectrum. Similar cases have been found in a few symbiotic X-ray binaries, where the hot component is $\sim 3$ orders of magnitude fainter than the red giant, and emission lines are absent in the optical spectra (Masetti et al. 2002, 2007).

In summary, DASCH J0757 is an intriguing system with many unusual properties. It differs from other known classic or symbiotic novae, and is more likely a new type of nova-like variable located in the gap region between classic novae and symbiotic novae. Unlike most other symbiotic stars, it does not contain a luminous hot component or nebula emission lines, and its magnitude is stable after the 1940s outburst. Its current photometric and spectroscopic profile is not different from a normal red giant binary. It would not be picked out without the detection of its long outburst in 1940s, made possible by the long timescales observed with DASCH. Future observation in the UV and X-ray will help understand its mass transfer and accretion processes.

Given the lack of other observations during the outburst of $\mathrm{J} 0757$, it is hard to reach a definite conclusion whether it was powered by hydrogen shell burning or accretion. If it was indeed hydrogen burning with no significant mass loss, it could be a promising channel of accumulating mass on the WDs. Systems similar to DASCH J0757, but with more massive WDs ( $\left.\gtrsim 1 M_{\odot}\right)$, could thus be promising Type Ia supernova (SN Ia) progenitors. They are not luminous supersoft X-ray sources in quiescence (see also Di Stefano 2010), and would not be picked out by the usual searches for symbiotic stars, but could be detected by the study of their long-term light curves. Note that J0757 is found from $\sim 700$ plates, while there are nearly half a million plates available to be scanned and analyzed in DASCH. With its 100 year light curve coverage, DASCH has a great potential to find more of such sources, which will shed light on the understanding of SN Ia progenitors, as well as the study of binary evolution and accretion physics.

We thank the anonymous referee for helpful comments. We are grateful to Alison Doane, Jaime Pepper, Edward Los, Robert J. Simcoe and David Sliski at CfA for their work on DASCH, and 
many volunteers who have helped digitize logbooks, clean, and scan plates (http://hea-www.harvard.edu/DASCH/team.php); and David Latham for his generous help on getting TRES spectra and data reduction. We thank Sumner Starrfield, Joanna Mikołajewska, Scott Kenyon, Mariko Kato, Lars Bildsten, Tony Piro, Sterl Phinney, Ken Shen, Maureen van den Berg, Rosanne Di Stefano, Jing Luan, and Perry Berlind for helpful discussions. S.T. thanks the FLWO staff and many visiting astronomers who acquired the TRES and FAST spectra; Andrea Dupree and Anna Frebel for the help on obtaining and reducing the MIKE spectra; Jessica Mink and Bill Wyatt for the initial reduction of the TRES and FAST spectra. This paper uses data products produced by the OIR Telescope Data Center, supported by the Smithsonian Astrophysical Observatory. This research has made use of the GSC 2.3.2, 2MASS, SDSS, and GALEX catalogs, HEASARC, NASA/IPAC Infrared Science Archive, and the ASAS database. This work was supported in part by the NSF grants AST0407380 and AST0909073 and now also the Cornel and Cynthia K. Sarosdy Fund for DASCH.

\section{REFERENCES}

Allen, D. A. 1980, MNRAS, 192, 521

Belczyński, K., Mikołjewska, J., Munari, U., Ivison, R. J., \& Friedjung, M. 2000, A\&AS, 146, 407

Bernstein, R. A., Shectman, S. A., Gunnels, S. M., Mochnacki, S., \& Athey, A. E. 2003, Proc. SPIE, 4841, 1694

Bertelli, G., Girardi, L., Marigo, P., \& Nasi, E. 2008, A\&A, 484, 815

Cox, A. N. 2000, Allen's Astrophysical Quantities (New York: Springer)

Di Stefano, R. 2010, ApJ, 712, 728

Downes, R. A., Duerbeck, H. W., \& Delahodde, C. E. 2001, J. Astron. Data, 7, 6

Eggleton, P. P. 1983, ApJ, 268, 368

Fabricant, D., Cheimets, P., Caldwell, N., \& Geary, J. 1998, PASP, 110, 79

Grindlay, J., Tang, S., Simcoe, R., et al. 2009, in ASP Conf. Ser. 410, Preserving Astronomy's Photographic Legacy: Current State and the Future of North American Astronomical Plates, ed. W. Osborn \& L. Robbins (San Francisco, CA: ASP), 101

Hauschildt, P. H., Allard, F., Ferguson, J., Baron, E., \& Alexander, D. R. 1999, ApJ, 525, 87

Iben, I., Jr. 2003, in ASP Conf. Ser. 303, Symbiotic Stars Probing Stellar Evolution, ed. R. L. M. Corradi, J. Mikołjewska, \& T. J. Mahoney (San Francisco, CA: ASP), 177

Kato, M., \& Hachisu, I. 1994, ApJ, 437, 802

Kato, M., Hachisu, I., Cassatella, A., \& González-Riestra, R. 2011, ApJ, 727, 72

Kenyon, S. J. 1986, The Symbiotic Stars (Cambridge: Cambridge Univ. Press) Kenyon, S. J. 1994, Mem. Soc. Astron. Ital., 65, 135

Kenyon, S. J., \& Webbink, R. F. 1984, ApJ, 279, 252

King, A. R., Rolfe, D. J., \& Schenker, K. 2003, MNRAS, 341, 35

Krautter, J., Woodward, C. E., Schuster, M. T., et al. 2002, AJ, 124, 2888
Kurucz, R. L. 2005, Mem. Soc. Astron. Ital., Suppl., 8, 10

Lasker, B. M., Sturch, C. R., McLean, B. J., et al. 1990, AJ, 99, 2019

Laycock, S., Tang, S., Grindlay, J., et al. 2010, AJ, 140, 1062

Lejeune, Th., Cuisinier, F., \& Buser, R. 1997, A\&AS, 125, 229L

Liller, M. H., \& Liller, W. 1979, AJ, 84, 1357

Los, E., Grindlay, J., Tang, S., Servillat, M., \& Laycock, S. 2011, in ASP Conf. Ser. 422, Astronomical Data Analysis Software Systems XX, ed. I. N. Evans, A. Accomazzi, D. J. Mink, \& A. H. Rots (San Francisco, CA: ASP), 269

Masetti, N., Dal Fiume, D., Cusumano, G., et al. 2002, A\&A, 382, 104

Masetti, N., Landi, R., Pretorius, M. L., et al. 2007, A\&A, 470, 331

Mikołajewska, J. 2003, in ASP Conf. Ser. 303, Symbiotic Stars Probing Stellar Evolution, ed. R. L. M. Corradi, R. Mikolajewska, \& T. J. Mahoney (San Francisco, CA: ASP), 9

Mikołajewska, J. 2007, Balt. Astron., 16, 1

Mikołajewska, J. 2011, The Proceedings of the Physics of Accreting Compact Binaries (Tokyo: Universal Academy Press)

Mikołajewska, J., Kolotilov, E. A., Shenavrin, V. I., \& Yudin, B. F. 2002, in ASP Conf. Ser. 261, The Physics of Cataclysmic Variables and Related Objects, ed. B. T. Gänsicke, K. Beuermann, \& K. Reinsch (San Francisco, CA: ASP), 645

Mikołajewski, M., Mikołajewska, J., \& Khudyakova, T. N. 1990, A\&A, 235, 219

Mink, D. J. 2011, in ASP Conf. Ser. 422, Astronomical Data Analysis Software Systems XX, ed. I. N. Evans, A. Accomazzi, D. J. Mink, \& A. H. Rots (San Francisco, CA: ASP), 305

Mürset, U., \& Nussbaumer, H. 1994, A\&A, 282, 586

Mürset, U., Nussbaumer, H., Schmid, H. M., \& Vogel, M. 1991, A\&A, 248, 458

Nomoto, K. 1982, ApJ, 253, 798

Nomoto, K., Saio, H., Kato, M., \& Hachisu, I. 2007, ApJ, 663, 1269

Nussbaumer, H. 2000, in ASP Conf. Ser. 204, Thermal and Ionization Aspects of Flows from Hot Stars: Observations and Theory, ed. H. Lamers \& A. Sapar (San Francisco, CA: ASP), 317

Orosz, J. A., \& Hauschildt, P. H. 2000, A\&A, 364, 265

Paczyński, B., \& Rudak, B. 1980, A\&A, 82, 349

Pickles, A. J. 1998, PASP, 110, 863

Podsiadlowski, P., \& Mohamed, S. 2007, Balt. Astron., 16, 26

Pojmanski, G. 2002, Acta Astron., 52, 397

Prialnik, D., \& Kovetz, A. 1995, ApJ, 445, 789

Schlegel, D. J., Finkbeiner, D. P., \& Davis, M. 1998, ApJ, 500, 525

Servillat, M., Los, E., Grindlay, J., Tang, S., \& Laycock, S. 2011, in ASP Conf. Ser. 422, Astronomical Data Analysis Software Systems XX, ed. I. N. Evans, A. Accomazzi, D. J. Mink, \& A. H. Rots (San Francisco, CA: ASP), 273

Shen, K., \& Bildsten, L. 2007, ApJ, 660, 1444

Skopal, A., Bode, M. F., Bryce, M., et al. 1996, MNRAS, 282, 327

Sokoloski, J. L., Kenyon, S. J., Espey, B. R., et al. 2006, ApJ, 636, 1002

Starrfield, S., Timmes, F. X., Iliadis, C., et al. 2012, Balt. Astron., 21, 75

Stasiǹska, G., \& Tylenda, R. 1990, in IAU Colloq. 122, Physics of Classical Novae, ed. A. Cassatella \& R. Viotti (Berlin: Springer), 293

Szentgyorgyi, A. H., \& Furész, G. 2007, Rev. Mex. Astron. Astrofis. Ser. Conf., 28,129

Tokarz, S. P., \& Roll, J. 1997, in ASP Conf. Ser. 125, Astronomical Data and Software Systems VI, ed. G. Hunt \& H. E. Payne (San Francisco, CA: ASP), 140

Townsley, D. M., \& Bildsten, L. 2004, ApJ, 600, 390

Zahn, J.-P. 1989, A\&A, 220, 112 